CASE ROPORT

\title{
A Case of Gluconacetobacter liquefaciens Bacteremia Associated with Sugarcane Juice Ingestion
}

\author{
Maxwell Olenski ${ }^{1,2}$, Lisa Brenton ${ }^{2}$, Lee Taylor ${ }^{2}$, Tim Papaluca ${ }^{3}$, Amy Crowe ${ }^{1,2}$ \\ ${ }^{1}$ Infectious Diseases Department, St Vincent's Hospital, Fitzroy, VIC, Australia \\ ${ }^{2}$ Microbiology Department, St Vincent's Hospital, Fitzroy, VIC, Australia \\ ${ }^{3}$ Gastroenterology Department, St Vincent's Hospital, Fitzroy, VIC, Australia
}

\begin{abstract}
Gluconacetobacter liquefaciens is a Gram-negative bacterium that has previously been described as a plant pathogen. To the best of our knowledge, only one previous case of human infection with Gluconacetobacter species has been described and was associated with severe immunodeficiency. We describe a case of recurrent Gluconacetobacter liquefaciens bacteremia in a patient with advanced liver cirrhosis and propose that gut translocation in the setting of portal hypertension and frequent sugarcane juice ingestion may have been the etiology. This case expands on our knowledge of Gluconacetobacter liquefaciens as a potential emerging human pathogen in certain situations and describes the difficulty encountered in antimicrobial testing and management. J Microbiol Infect Dis 2020; 10(1):62-67.
\end{abstract}

Keywords: Environmental, Alphaproteobacteria, Gluconacetobacter liquefaciens, Gram-negative, Bacteremia, Cirrhosis

\section{INTRODUCTION}

Gluconacetobacter species are non-endospore forming obligate aerobic Gram-negative bacteria which are ellipsoidal or rod-shaped, and measure 0.6-1.2 x 1.0-3.0 $\mu \mathrm{m}$ [1]. They occur singly, in pairs or short chains, and have both motile and non-motile forms.

Growth is achieved at $30{ }^{\circ} \mathrm{C}$ with a pH between 2.5-6.0 utilizing various media, including Orange Serum agar which is used for isolation, cultivation and enumeration of acid-tolerant spoilage microorganisms in fruit juice and citrus products [2]. It utilizes ethanol, glucose and acetate for growth, and, biochemically, is catalase positive but oxidase, indole, and $\mathrm{H}_{2} \mathrm{~S}$ negative [3].

Gluconacetobacter species can cause spoilage of beer, wine and spirits, yet play an integral role in vinegar production. In addition, they can contaminate kombucha and sugarcane [3].

Gluconacetobacter liquefaciens was first described by Asai in 1935 [4], and though the biology of acetic acid bacteria is well described in production and spoilage in the food and beverage industry, blood stream infection from
Gluconacetobacter species has never been described in humans nor animals; this may be attributable to difficulties encountered isolating and identifying this bacterium, which may have resulted in previous cases being overlooked.

We describe a case of recurrent $G$. liquefaciens bacteremia in the context of cirrhosis with portal hypertension.

\section{CASE REPORT}

A 48-year-old male was admitted to a tertiary referral center with a brief history of acute on chronic right upper quadrant abdominal pain and fever with associated decompensated liver disease in October 2019.

The patient had regular review in Gastroenterology outpatients for management of compensated chronic liver disease. He was initially referred with Child Pugh C decompensated chronic liver disease in February 2017 in the setting of alcohol dependence, chronic hepatitis $\mathrm{C}[\mathrm{HCV}]$ and chronic hepatitis $B$ [CHB] infection. His HCV was treated with 24 weeks of sofosbuvir and daclatasvir with sustained virological response. 
He commenced tenofovir disoproxil fumarate [TDF] for CHB infection and has maintained sobriety for greater than two years. In this setting, he recompensated to Child Pugh A status. He has comorbid hypertension and cholelithiasis. In addition to TDF, his medications included antihypertensives, a proton pump inhibitor and regular aperients. He is a regular smoker and previously identified as a person who injects drugs. He had chronic right upper quadrant pain which was thought to relate to capsular stretch from hepatomegaly. There were no hepatic vascular abnormalities nor infiltrative processes on imaging.

On initial presentation to the Emergency Department, the patient reported a two-day history of acute on chronic right upper quadrant pain and abdominal distention. Prior to this, he had undergone workup for elective cholecystectomy including upper abdominal ultrasonography and magnetic retrograde cholangiopancreatography; this demonstrated cholelithiasis, as well as dilated intra- and extrahepatic ducts which tapered normally at the Ampulla of Vater. There was no evidence of choledocholithiasis.

On examination, the patient was normotensive but febrile with a temperature of $38.6^{\circ} \mathrm{C}$. There were no abnormalities identified on cardiorespiratory examination, and he had tender hepatomegaly without demonstrable peritonism. With a presumed diagnosis of acute cholecystitis, he was commenced on intravenous ceftriaxone and admitted under the Hepatobiliary Unit.

Baseline bloodwork was significant for a microcytic anemia with a hemoglobin of $71 \mathrm{~g} / \mathrm{L}$, a normal white cell count of $6.0 \times 10^{9} / \mathrm{L}$, and acutely worsened thrombocytopenia (platelets $\left.44 \times 10^{9} / \mathrm{L}\right)$. Biochemistry revealed stably deranged liver function tests, with an alkaline phosphatase of $149 \mathrm{U} / \mathrm{L}$, a gamma-glutamyl transferase of $126 \mathrm{U} / \mathrm{L}$ and a normal alanine aminotransferase of $14 \mathrm{U} / \mathrm{L}$. Baseline C-reactive protein [CRP] was $20 \mathrm{mg} / \mathrm{L}$. His albumin had acutely reduced to $25 \mathrm{~g} / \mathrm{L}$, having been $36 \mathrm{~g} / \mathrm{L}$ three weeks prior, and his renal function was normal.

Upper abdominal ultrasonography revealed cholelithiasis with a thickened gallbladder wall but neither hyperemia nor probe-tenderness.
Antibiotics were broadened with the addition of metronidazole.

Three weeks prior to this presentation the patient had undergone transjugular wedged portal venous pressure measurement to prognosticate his surgical risk and appropriateness for cholecystectomy. A percutaneous liver biopsy was also performed to exclude infiltrative pathologies contributing to his chronic right upper quadrant pain and hepatomegaly. The hepatic venous portal gradient confirmed significant portal hypertension, and the biopsy showed micronodular cirrhosis and mild inflammation. There were no periprocedural complications.

After three days of incubation and monitoring in an automated blood culture system (BD BactecTMFX ), blood cultures drawn on the first day of fever flagged positive in the aerobic bottle. Review of the Gram stain revealed elongated, non-branching, thick Gram-negative bacilli (Figure 1A).

Incubation in aerobic conditions at $37^{\circ} \mathrm{C}$ for two days revealed no observable growth on Chocolate, MacConkey nor Trypticase soy agar with sheep blood (TSA+SB) (Thermo Fisher Scientific, Australia); likewise, there was no growth under anaerobic conditions. After a further day of incubation, scant growth was observed on Chocolate agar. Subsequently, the organism was identified to genus level using Matrix-Assisted Laser Desorption/IonizationTime of Flight (MALDI-TOF, Bruker, Germany) mass spectrometry as Gluconoacetobacter spp with a probability score of 1.74 , which was felt to represent environmental contamination.

Over the ensuing weeks of admission, the patient continued to have recurrent fevers in the absence of an identifiable focus for infection, during which time he remained clinically well. Three subsequent aerobic blood cultures flagged positive for Gram-negative bacilli. This time, faint growth was observed on both Chocolate agar and TSA+SB incubated aerobically at $37^{\circ} \mathrm{C}$ for three days (Figure $1 \mathrm{~B}$ ). On suspicion that the organism was a Gramnegative bacillus of environmental origin, it was incubated aerobically at $30^{\circ} \mathrm{C}$ on Chocolate agar and TSA+SB. After a further three days, medium-sized, cream-colored colonies with entire margins were observed (Figure 1C). 
Biochemical testing revealed a catalase positive, oxidase and indole negative organism. An API 20NE demonstrated D-glucose assimilation but was negative for glucose fermentation.

At this time, the isolate was again identified as Gluconacetobacter spp (probability score of 1.94) by MALDI-TOF. Turbidity and pellicle formation were present in thioglycolate broth (Figure 2). Owing to its fastidious nature, and the relative obscurity of this organism, the isolate was sent to a reference laboratory for 16S rRNA testing. Subsequently, this matched with Gluconoacetobacter liquefaciens in the Genbank database with a reported $99.91 \%$ similarity.

Orange Serum agar (Thermo Fisher, Scientific, Australia) was sourced by our laboratory. The organism grew best on this media incubated aerobically at $30^{\circ} \mathrm{C}$, with Gram's stain revealing short Gram-negative bacilli (Figure 1D), and growth of medium-sized cream and pink colonies.

Attempts at susceptibility testing were conducted on Mueller-Hinton agar with $5 \%$ sheep blood using E-tests after lawn culture. Minimum inhibitory concentrations [MICs] were read after 24 hours of incubation at $30^{\circ} \mathrm{C}$ in aerobic conditions with the following results: ciprofloxacin (MIC $\geq 32 \mathrm{ug} / \mathrm{mL}$ ), ceftriaxone (MIC $=12 \mathrm{ug} / \mathrm{mL}$ ), meropenem (MIC $\geq 32 \mathrm{ug} / \mathrm{mL}$ ), gentamicin ( $\mathrm{MIC}=0.125 \mathrm{ug} / \mathrm{mL}$ ), tetracycline $(\mathrm{MIC}=1 \mathrm{ug} / \mathrm{mL})$, cotrimoxazole $(\mathrm{MIC} \geq 32$ $\mathrm{ug} / \mathrm{mL}$ ) and chloramphenicol (MIC $\geq 256 \mathrm{ug} / \mathrm{mL}$ ). At 48 hours of incubation, ceftriaxone showed an MIC of $\geq 32 \mathrm{ug} / \mathrm{mL}$, and gentamicin showed an MIC of $0.25 \mathrm{ug} / \mathrm{mL}$, whereas tetracycline retained an $\mathrm{MIC}$ of $1 \mathrm{ug} / \mathrm{mL}$.

Throughout this period, the patient remained clinically stable apart from intermittent fevers. These were not accompanied by systemic upset, with a CRP continuing to downtrend following admission and institution of antimicrobial therapy, which was adjusted to meropenem prior to the results of 16S rRNA testing becoming available.

On further questioning, the patient reported twice-weekly ingestion of freshly pressed sugarcane juice obtained from a local juice bar. In addition, he reported no kombucha consumption, and reported abstinence from both alcohol and intravenous drug use.
Over the subsequent days his fever abated, and his abdominal pain settled. Investigation for a deep-seated focus of infection was noncontributory, with computed tomography revealing no intra-abdominal collections nor ascites, and Fluorodeoxyglucose Positron Emission Tomography not suggestive of an occult nidus of infection. Transthoracic echocardiography showed mildly increased basal septal wall thickness with left ventricular outflow obstruction, however, did not reveal any independently mobile echogenicities.

Upon review of antimicrobial susceptibilities, he was discharged with six-weeks of twice-daily oral doxycycline. He awaits repeat outpatient transthoracic echocardiography, clearance blood cultures following cessation of antibiotics, after which liver transplant appropriateness will be considered.

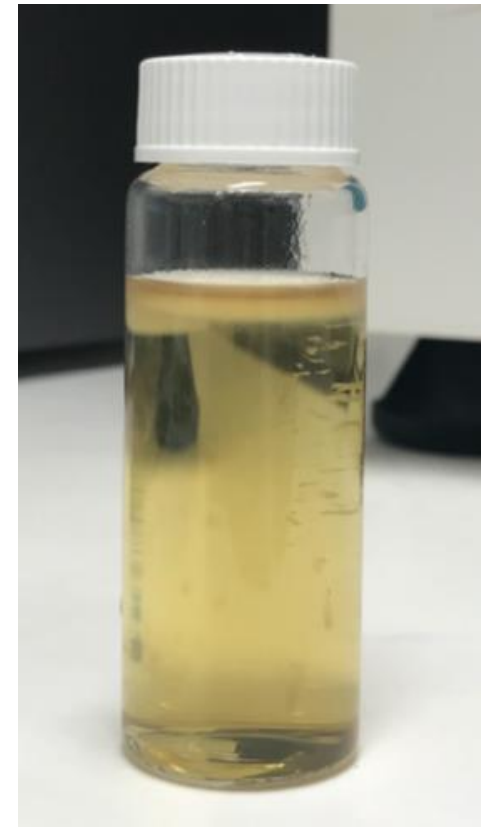

Figure 2. Pellicle formation in thioglycolate broth after 48 hours

\section{DISCUSSION}

Amongst the Proteobacteria phlya, Gluconacetobacter spp are phylogenetically distinct. Whereas commonly encountered Gramnegative bacteria - such as those from the family Enterobacterales - derive from Gammaproteobacteria, Gluconacetobacter spp are classified amongst Alphaproteobacteria and belong to the family Acetobacteraceae, bacteria that are found widely in the environment [5]. 


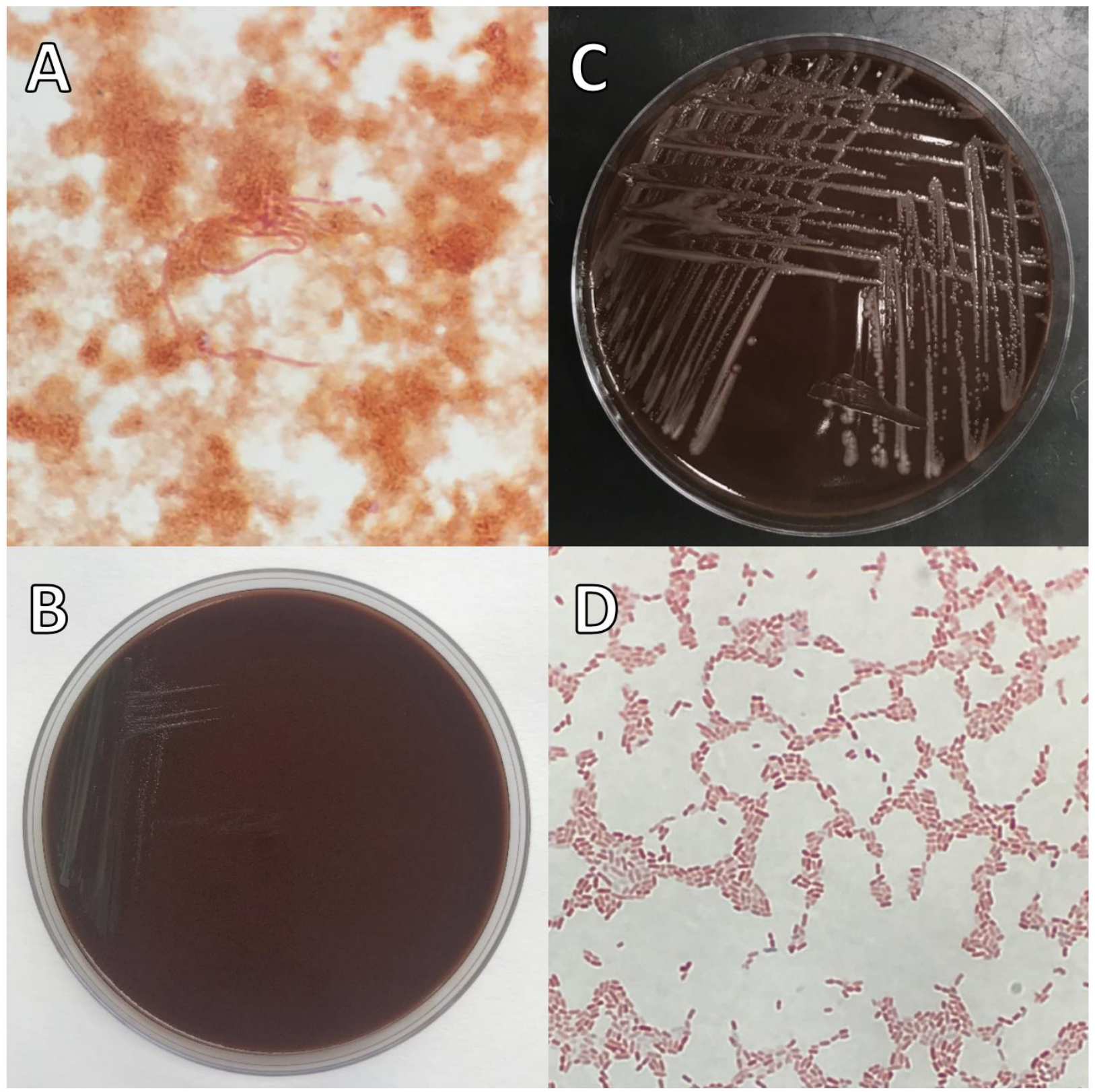

Figure 1. Panel A shows the initial Gram stain from aerobic blood culture incubated for 72 hours. Panel B shows colony growth on Chocolate agar incubated aerobically at $37^{\circ} \mathrm{C}$ after 72 hours. Panel $\mathrm{C}$ show colony growth on Chocolate agar incubated aerobically at $30^{\circ} \mathrm{C}$ after 48 hours. Panel D shows the Gram stain of colonies isolated on Orange Serum agar.

Whilst generally considered an organism of environmental origin, given repeated isolation in blood from our patient, as well as a curious epidemiological link in the form of sugarcane ingestion, we postulate that Gluconoacetobacter liquefaciens infection drove these recurrent fevers. Inadvertent direct inoculation of the organism in the context of intravenous drug use remains a consideration, though this was not gleaned historically.

Greenberg et al describe a case of lymphadenitis associated with Gluconacetobacter sacchari infection in the context of host immune dysfunction related to Chronic Granulomatous Disease [6]. To the best of our knowledge, this is the first case in which 
this Gluconacetobacter liquefaciens has been described as a causative agent of disease.

There are no Clinical and Laboratory Standards Institute or European Committee on Antimicrobial Susceptibility Testing interpretative criteria for antimicrobial susceptibility reporting of Gluconacetobacter spp. Given the propensity for $\beta$-lactam resistance in similar Gram-negative bacteria in the Acetobacteraceae family [7], in conjunction with our antimicrobial susceptibility data, we elected to alter therapy to doxycycline.

We propose that host factors rendered the patient prone to infection. Several studies show that cirrhosis predisposes to the development of bacterial overgrowth within the bowel, as well as increased intestinal permeability and resultant bacterial translocation [8-10]. This effect may be compounded by concomitant use of proton pump inhibitors, which results in hypochloridria and may favor bacterial proliferation [11]. In addition, bacteria within the bowel lumen can colonize mesenteric lymph nodes through migration across the bowel wall, and portal hypertension can result in both bacterial contamination of ascitic fluid from ruptured infected lymphatic channels, as well as translocation directly into the systemic circulation, which in turn can result in bacteremia.

Cirrhosis-associated immune dysfunction [CAID], too, is a dynamic phenomenon, resulting in perturbations of both innate and acquired immunity [12]. Increased anti-inflammatory cytokines and suppression of proinflammatory cytokines, in conjunction with enhanced bowel permeability and bacterial translocation, predisposes to endotoxemia, bloodstream infection, sepsis, multiorgan failure and death [13].

\section{Conclusion}

Acetobacteraceae are organisms frequently encountered in the environment but have seldom been reported to cause invasive infection. This, perhaps, relates to difficulty in culturing this organism from pathologic specimens.

Given the association between Gluconacetobacter spp and our patient's regular sugarcane juice consumption, in the context of his underlying cirrhosis, portal hypertension and ensuing CAID, we surmise that repeated ingestion of the organism resulted in his bloodstream infection. Whilst it is unclear whether repeated isolation of this bacterium from the blood represents relapse, reinfection or an unidentified reservoir, is clear is that this pathogen is of low virulence.

Further research into the role of such organisms in human disease is warranted.

\section{ACKNOWLEDGMENTS}

Declaration of Conflicting Interests: The authors declare that they have no conflict of interest.

Financial Disclosure: No financial support was received.

\section{REFERENCES}

1. Sievers M, Swings J. Gluconacetobacter. In: Bergey's Manual of Systematics of Archaea and Bacteria. John Wiley \& Sons, Ltd; 2015. p. 1-11.

2. Oxoid Limited. Oxoid - Product Detail [Internet]. [cited 2019 Nov 26]. Available from: http://www.oxoid.com/UK/blue/prod_detail/prod_d etail.asp?pr=CM0657\&org=82\&C=UK\&lang=EN

3. Swings J, De Ley J. The Genera Gluconobacter and Acetobacter. In: The Prokaryotes. Springer Berlin Heidelberg; 1981. p. 771-8.

4. ASAI, T. Taxonomic studies on acetic acid bacteria and allied oxidative bacteria isolated from fruits. A new classification of the oxidative bacteria. J Agric Chem Soc Japan. 1935; 11:674708.

5. NCBI taxonomy database. Taxonomy browser (Gluconacetobacter) [Internet]. [cited 2019 Dec 26]. Available from: https://www.ncbi.nlm.nih.gov/Taxonomy/Browser/ wwwtax.cgi? mode $=$ Info $\& i d=89583 \&|v|=3 \&$ lin $=f \& k e$ ep $=1$ \&srchmode $=1$ \&unlock

6. Greenberg DE, Ding L, Zelazny AM, et al. A novel bacterium associated with lymphadenitis in a patient with chronic granulomatous disease. PLoS Pathog [Internet]. 2006 [cited 2019 Nov 14];2(4):260-7. Available from: www.plospathogens.org

7. Cools $P$, Nemec A, Kampfer $P$, Vaneechoutte $M$. Acinetobacter, Chryseobacterium, Moraxella, and Other Non-Fermentative Gram-Negative Rods. In: Manual of Clinical Microbiology, 12th Edition. 2019. p. 829-57.

8. Cirera I, Martin Bauer T, Miguel N, et al. Bacterial translocation of enteric organisms in patients with cirrhosis. J Hepatol. 2001;34(1):32-37.

9. Scarpellini E, Valenza V, Gabrielli M, et al. Intestinal permeability in cirrhotic patients with and 
without spontaneous bacterial peritonitis: is the ring closed? Am J Gastroenterol [Internet]. 2010 Feb [cited 2019 Nov 21];105 (2):323-7. Available from:

http://www.ncbi.nlm.nih.gov/pubmed/19844200

10. Runyon BA, Squier $S$, Borzio $M$. Translocation of gut bacteria in rats with cirrhosis to mesenteric lymph nodes partially explains the pathogenesis of spontaneous bacterial peritonitis. J Hepatol. 1994;21(5):792-796.

11. Sánchez E, Soriano G, Mirelis B, Gonzalez B, Nieto JC, Vidal S, et al. Effect of long-term acid gastric inhibition on bacterial translocation in cirrhotic rats. Eur J Gastroenterol Hepatol 2015 27(5):570-6.

12. Talha Noor M, Manoria P. Citation of this article: Noor MT, Manoria P. Immune dys-function in cirrhosis. J Clin Transl Hepatol [Internet]. 2017 [cited 2019 Dec 17];5(1):50-8. Available from: http://www.jcthnet.com

13. Clària J, Mitchell DA, Irvine KM, Ratnasekera I, Powell EE, Hume DA. Causes and Consequences of Innate Immune Dysfunction in Cirrhosis. Front Immunol | www.frontiersin.org [Internet]. 2019 [cited 2019 Dec 17]; 10:293. Available from: www.frontiersin.org 